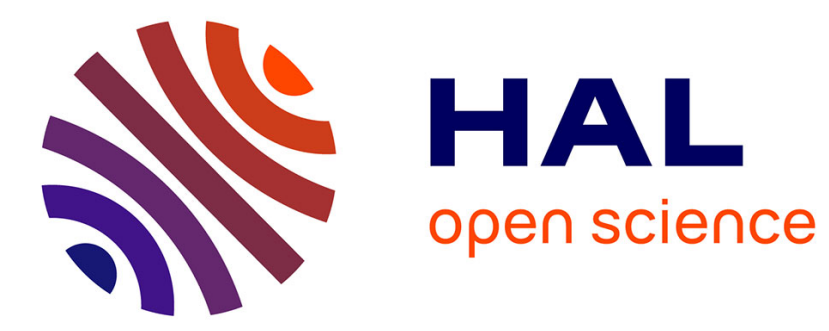

\title{
Display calculi and other modal calculi: a comparison
} Francesca Poggiolesi

\section{To cite this version:}

Francesca Poggiolesi. Display calculi and other modal calculi: a comparison. Synthese, 2010, 173 (3), pp.259-279. 10.1007/s11229-008-9425-4 . halshs-00775789

\section{HAL Id: halshs-00775789 \\ https://shs.hal.science/halshs-00775789}

Submitted on 4 Jul 2018

HAL is a multi-disciplinary open access archive for the deposit and dissemination of scientific research documents, whether they are published or not. The documents may come from teaching and research institutions in France or abroad, or from public or private research centers.
L'archive ouverte pluridisciplinaire HAL, est destinée au dépôt et à la diffusion de documents scientifiques de niveau recherche, publiés ou non, émanant des établissements d'enseignement et de recherche français ou étrangers, des laboratoires publics ou privés. 


\title{
Francesca Poggiolesi Display calculi and other modal calculi: a comparison
}

\author{
In view of the diversity of these types \\ of proof systems it becomes increasingly \\ important to investigate their interrelations \\ and their advantages and disadvantages. \\ Wansing (1998), p. 171
}

\begin{abstract}
In this paper we introduce and compare four different syntactic methods for generating sequent calculi for the main systems of modal logic: the multiple sequents method, the higher-arity sequents method, the treehypersequents method and the display method. More precisely we show how the first three methods can all be translated in the fourth one. This result sheds new light on these generalisations of the sequent calculus and raises issues that will be examined in the last section.
\end{abstract}

\section{Introduction}

Since the 80's, research in proof theory for modal logic (and other non-classical logics) has led to the development of several generalisations of the original Gentzen calculus. These generalisations can be divided in two groups: in one group we have extensions of the sequent calculus that explicitly use semantic elements, such as variables ranging over possible worlds, while in the other group we have purely syntactic extensions of the sequent calculus. This is a first distinction that we can make. But we can also make another one. We can divide the generalisations of the classical sequent calculus for modal logic into those which have only been applied to one or two modal systems, e.g. the system $\mathbf{S} \mathbf{4}$ or the system S5, and those which, by contrast, generate calculi for a wide range of modal systems. In this paper we only take into account those generalisations that are purely syntactic and that concern a range of modal systems. We underline that this choice is not arbitrary, on the contrary it is well-motivated. Indeed we want to deal with good and strong Gentzen systems: therefore we rule out those calculi that use semantic elements, since we cannot really consider them to be proof-theoretical instruments (e.g. see Avron (1996)), and we concentrate on those that have been applied to several modal systems so that we can be sure of their efficacy and generality.

The starting point of each of the generalisations of the Gentzen calculus that we are going to take into account in this paper is a question that we can naturally ask concerning a classical sequent: is it possible to find a more abstract version of this kind of object? The answer is not only affirmative, but there are several ways of providing it. Here we only consider four of them: 
- following Indrezejczak (1997) who has introduced the multiple sequent calculi, we can deal with more than just one type of consequence relation;

- following Blamey and Humberstone (1991) who have introduced the higherarity sequent calculi, we can deal with more than just one antecedent and one succedent;

- following Kashima (1994) and Poggiolesi (2008b), who have introduced the tree-hypersequent calculi, we can deal with $n$ different sequents a time arranged in such a way that we can simulate a tree-shape;

- following Belnap (1982) and Wansing (1994) who have introduced the display calculi, we can deal with several meta-linguistic symbols.

Our goal in this paper is to show the relationships between these generalisations. More particularly we will show that multiple sequents, higher-arity sequents and tree-hypersequents can be simulated by display sequents. This fact reveals the great expressive power of the display method and raises issues that we shall examine in the last section. We conclude the introduction by emphasising that the problem of the relationships between different types of modal calculi has already been treated by, as far as we know, four other authors: Wansing (1998) who has shown how to simulate hypersequents by display sequents; Mints (1997) who, by contrast, has proved that we can plug indexed sequent calculi in display calculi; and, finally, Goré and Tiu (2007) who have shown that we can plug display calculi in the calculi of structures. Note that the calculus of structures (e.g. see Stewart and Stouppa (2005), Guglielmi (2007)) is a generalisation of the sequent calculus rather different from the ones that we have presented above: it is not obtained by introducing a more abstract version of the notion of sequent, but by changing the structure of the sequent calculus itself. All these authors, Goré, Mints, Tiu and Wansing, have emphasised the importance of investigating the problem of the relationships between different types of modal calculi, since it serves to shed light on the advantages, the peculiarities and the proper workings of each proof method.

\section{Display Calculi}

The term display logic is usually used to refer to a general proof theoretic schema introduced by Belnap (1982) (see also Belnap (1990), Belnap (1996)). This schema has been fully exploited not only in the field of modal logic, where the work of Wansing (1994) (see also Wansing (2002), Wansing (1998)) stands out, but also in other fields such as substructural logics, (Goré (1998), Restall (1998)), or the one of subintuitionistic logic (Wansing (1997)). The basic idea of display logic is to start looking at the sequent arrow as a deducibility relation between finite possible complex data. In line with this interpretation, one does not work any more with finite multisets, or sets of formulas, as in the classical 
Figure 1: Axioms and Structural Rules of the Display Calculi.

$$
\begin{aligned}
& p \Rightarrow p \quad \frac{M \Rightarrow N}{I \circ M \Rightarrow N} I+\quad \frac{M \Rightarrow N}{M \circ I \Rightarrow N}{ }^{I+} \quad \frac{I \circ M \Rightarrow N}{M \Rightarrow N}{ }_{I-} \\
& \frac{M \circ I \Rightarrow N}{M \Rightarrow N} I_{-} \quad \frac{I \Rightarrow N}{M \Rightarrow N} I A \quad \frac{M \Rightarrow I}{M \Rightarrow N} I K \quad \frac{M_{1} \circ\left(M_{2} \circ M_{3}\right) \Rightarrow N}{\overline{\left(M_{1} \circ M_{2}\right) \circ M_{3} \Rightarrow N}} A \\
& \frac{M_{1} \circ M_{2} \Rightarrow N}{M_{2} \circ M_{1} \Rightarrow N} P \quad \frac{M \circ M \Rightarrow N}{M \Rightarrow N} C \quad \frac{I \Rightarrow N}{\bullet I \Rightarrow N} r n \quad \frac{M \Rightarrow \alpha \quad \alpha \Rightarrow Q}{M \Rightarrow Q} C_{C u t_{\alpha}}
\end{aligned}
$$

Figure 2: Basic Structural Rules of the Display Calculi.

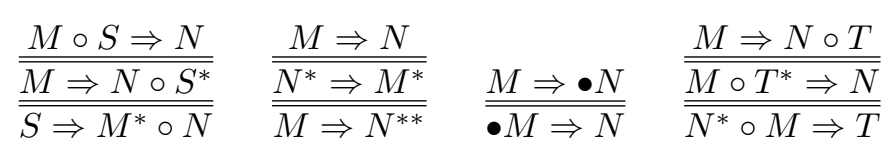

sequent calculus, but starts to deal with the so called Gentzen terms or structures, and adds new structural symbols that are applicable to such Gentzen terms or structures. Thanks to these innovations it becomes possible to simulate, in the framework of the sequent calculus, the most natural and desirable data-operations that we can think of: e.g. combining the data, transferring the data, moving it around.

Syntactic Notation. Beyond the rightarrow symbol, we employ the following four new structural connectives: $I$ : a nullary operation; $\bullet, *$ : unary operations; $\circ$ : a binary operation. Intuitively these structural connectives should be understood in the following way: the symbol $I$ should be seen as the empty structure; the symbol $\circ$ as the structure addition that replaces the comma and that should be read as a conjunction if situated on the left side of the sequent, while as a disjunction if situated on the right side of the sequent; the symbol $*$ as the operator that shifts structures from one side to the other; the symbol $\bullet$ as the operator that marks the structure in its scope as intensional.

Given that the set of well-formed modal formulas is defined in the standard way, a structure in display logic is given by the rule:

$$
M::=I|\alpha| \bullet M\left|M^{*}\right| M \circ N
$$

Therefore every modal formula $\alpha$ is considered as a structure and the structural connectives are used to build up more complex structures in the obvious way.

Display Sequent. Let us assume that $M, N, \ldots$ vary on structures. Then a sequent in display calculi is an object of the form $M \Rightarrow N$. The structure $M(N)$ is the antecedent (succedent) of $M \Rightarrow N$. The structure $M$ occurs negatively 
Figure 3: Logical and Modal Rules of the Display Calculi

$$
\begin{array}{rlrl}
\frac{\alpha^{*} \Rightarrow N}{\neg \alpha \Rightarrow N} \neg A & \frac{M \Rightarrow \alpha^{*}}{M \Rightarrow \neg \alpha} \neg K & \frac{\alpha \circ \beta \Rightarrow N}{\alpha \wedge \beta \Rightarrow N} \wedge A^{\prime} & \frac{M \Rightarrow \alpha \quad P \Rightarrow \beta}{M \circ P \Rightarrow \alpha \wedge \beta} \wedge K^{\prime} \\
& \frac{\alpha \Rightarrow N}{\square \alpha \Rightarrow \bullet N} \square A & \frac{\bullet M \Rightarrow \alpha}{M \Rightarrow \square \alpha} \square K
\end{array}
$$

Figure 4: Special Structural Rules of the Display Calculi

$$
\begin{aligned}
& \frac{\bullet M \circ \bullet N \Rightarrow I^{*}}{M \Rightarrow N^{*}} d \quad \quad \frac{M \Rightarrow \bullet N}{M \Rightarrow N} t_{t} \quad \frac{M \Rightarrow \bullet N}{M \Rightarrow \bullet \bullet N}{ }^{4} \quad \frac{\left(\bullet\left(M^{*}\right)\right)^{*} \Rightarrow N}{\bullet M \Rightarrow N}{ }_{b} \\
& \frac{\left(\bullet\left(M^{*}\right)\right)^{*} \Rightarrow N}{\bullet\left(\left(\bullet\left(M^{*}\right)\right)^{*}\right) \Rightarrow N^{5}}
\end{aligned}
$$

in $M^{*}$, and occurs positively in each of $\bullet M$ and $(M \circ N)$ and $(N \circ M)$. A substructure $P$ is an antecedent (succedent) part of a sequent $M \Rightarrow N$, if it occurs positively (negatively) in $M$ or negatively (positively) in $N$.

Display Equivalence, Display Property and Cut-elimination. Let us concentrate on the basic structural rules of the display calculi (see Figure 2). These rules determine the simple and clear inferential behaviour of the four new structural connectives. For example the first two triplets of rules show how the two structural connectives $\circ$ and $*$ (the addition of data and their transfer, respectively) interact together. Notice that if two sequents are interderivable by means of the basic structural rules, then these sequents are said to be structurally equivalent.

One of the crucial features of display logic, by which it takes its name, is the display theorem, that, expressed informally, claims that any substructure of a given display sequent $s$ may be displayed as the entire antecedent or succedent, respectively, of a structurally equivalent sequent $s^{\prime}$. More precisely the display theorem states that:

Theorem 2.1. For every display sequent $s$ and every antecedent (succedent) part $M$ of $s$, there exists a sequent s' structurally equivalent with $s$, such that $M$ is the antecedent (succedent) of $s^{\prime}$.

If a logic satisfies the Display Theorem is said to satisfy the display property. As remarked by Wansing (1998, p. 36) the basic structural rules that we have given above suffice to prove the Display Theorem, but there exist other combinations of basic structural rules that guarantee the display property. Let us finally remark that the display theorem has an important technical significance: it allows an elegant and uniform proof of the cut-elimination theorem.

Logical and Modal Rules The logical and modal rules of the display calculi 
(see Figure 3) maintain the display property since the formula they introduce represents either the whole antecedent or the whole consequent of the sequent. We assume the symbols $\neg, \wedge$ and $\square$ as primitive, the others can be defined as usual.

Modularity. The axioms, the structural rules, the basic structural rules, the logical and modal rules compose the display calculus Dsk for the basic modal system K. In order to obtain the calculi for the other modal systems, we just add the appropriate special structural rule(s) (see Figure 4). Indeed, as is evident from their names, each special structural rule corresponds to one of the modal axioms $D, T, 4, B, 5$, and reflects it at the proof-theoretical level. This correspondence takes the name of modularity and represents one of the attractive characteristics of display logic.

Two Other Properties of the Display Calculi. There are many results provable in the display framework. Here we quote just two that will prove useful later: (i) the axioms of the form $\alpha \Rightarrow \alpha$ are admissible in the display calculi; (ii) the logical rules and the modal rule $\square K$ can be proved to be invertible with the use of the cut-rule.

\section{Multiple Sequent Calculi and Display Calculi}

The multiple sequent calculi, introduced by Indrezejczak (1997), are based on the idea, which seems to go back to Curry (1952) and Zeman (1973), of dealing with two different types of consequence relation: the normal one $(\Rightarrow)$ and the modal one $(\square \Rightarrow)$. Intuitively the difference between these two types of consequence relation can be explained in the following way: a classical sequent is said to be unsatisfiable if, simply, the antecedent is true and the consequent false. The same holds for modal sequents even if, in this case, we have to make reference to two different worlds of a Kripke model: the antecedent is true in one, and the consequent false in the other.

Syntactic Notation. First of all we denote the standard modal language restricted to the connectives $\neg, \wedge, \square$ with the notation $\mathcal{L}^{\square}\{\neg, \wedge, \square\}$, and the set of well-formed formulas, defined in the usual way, with the notation $W M F$. Then let $\mathcal{L}_{-}^{\square}$ be the language that extends $\mathcal{L}^{\square}\{\neg, \wedge, \square\}$ by adding the unary connective "-". Any $\alpha \in W M F$ may be prefixed with the symbol - . This symbol cannot be nested or iterated: things like $-\alpha$ or $\alpha \wedge-(\beta \vee-\gamma)$ are not well-formed formulas. Let us therefore define the set of well-formed formulas $W M F_{-}$of the language $\mathcal{L}_{-}^{\square}$ in the following way: $W M F_{-}:=W M F \bigcup\{-\alpha \mid \alpha \in W M F\}$. Moreover we have:

$$
\text { for every } \alpha \in W M F_{-}, \alpha^{*}= \begin{cases}\beta, & \text { if } \alpha \equiv-\beta \\ -\alpha, & \text { otherwise. }\end{cases}
$$

Hence we also have: $M^{*}:=\{\alpha \mid-\alpha \in M\} \cup\{-\alpha \mid \alpha \in M\}$. Finally we call $B$-formulas any $\square \alpha$ or $\square \neg \alpha$ in the antecedent of a sequent. Accordingly $B[M]$ stands for: the multiset $M$ is composed of $B$-formulas. 
Figure 5: Axioms and Structural Rules of the Multiple Sequent Calculi

$$
\begin{aligned}
& \alpha \Rightarrow \alpha \quad \frac{M \underset{n}{\rightleftarrows} N}{\alpha, M \underset{n}{\rightleftarrows} N} W A \quad \frac{M \underset{n}{\rightleftarrows} N}{M \underset{n}{\Rightarrow} N, \alpha} W K \quad \frac{\alpha, \alpha, M \underset{n}{\Rightarrow} N}{\alpha, M \underset{n}{\Rightarrow} N} C A \\
& \frac{M \underset{n}{\rightrightarrows} N, \alpha, \alpha}{M \underset{n}{\rightrightarrows} N, \alpha} C K \quad \frac{\Rightarrow N}{\square N} \text { rn }
\end{aligned}
$$

Figure 6: Shifting Rules of the Multiple Sequent Calculi

$$
\frac{M \Rightarrow N, \alpha}{\alpha^{*}, M \Rightarrow N} S A \quad \frac{\alpha, M \Rightarrow N}{M \Rightarrow N, \alpha^{*}} S K
$$

Multiple sequent. Given two $W M F_{-}$multisets $M$ and $N$, a modal sequent is an object of the following form:

$$
M \square \underset{n}{\Rightarrow} N
$$

which stands for: $M \overbrace{\square . . . \square}^{n} \Rightarrow N$. In the calculi for the modal systems containing the axiom $B, n=0,1, \ldots$, in the rest of the calculi $n=0,1$. Clearly if $n=0$, then $\Rightarrow$ is just a classical sequent and we will write as usual $M \Rightarrow N$. Notice that the modal sequent arrow $\square \Rightarrow \vec{n}$ fails to represent a consequence relation because (in general) $\alpha \underset{n}{\Rightarrow} \alpha$ does not hold.

Structural and Shifting Rules. We would like to point out two features of the the multiple sequent calculi. The first and more evident is the lack of a cut-rule (see Figure 5). The second is the presence of shifting rules (see Figure 6) that make use of the connective "-" and that shift, anagously to the basic structural rules of the display calculi, a formula $\alpha$ from one side of the sequent to the other, i.e., they do what in the classical sequent calculus is part of the "job" of the logical rules for the connective $\neg$ (and also of the logical rules for the connective $\rightarrow$, if it is taken as primitive). The distinction between shifting rules and logical rules has been firstly introduced by Fitting (1983) in order to prove the interpolation theorem; here we use it to ensure that the logical rules can be applied to any type of sequent: the classical one $(n=0)$ and modal one $(n>0)$.

Logical and Modal Rules. The logical rules for the symbol $\neg$ and $\wedge$ are straightforward adaptations of the classical rules to the multiple case (and this is the reason why we do not even state them explicitly). Notice only that: (i) they hold for any type of sequent: the classical one $(n=0)$ and the modal one $(n>0)$; (ii) in case of a two premise rules, the two premises should involve the same type of sequent. The modal rules are the following: 
Figure 7: Special Structural Rules of the Multiple Sequent Calculi

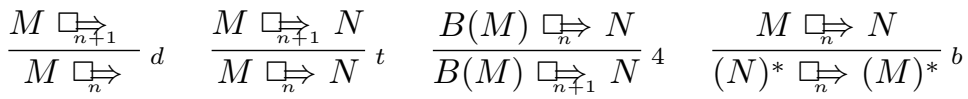

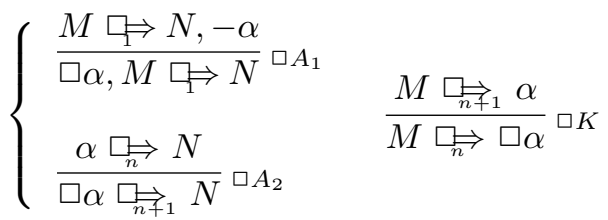

Modularity. The axioms, the structural rules, the shifitng rules, the logical and modal rules compose the calculus Msk for the basic modal system K. In order to obtain the calculi for other modal systems, we just add the appropriate special structural rule(s) (see Figure 7), as we have explained for the display calculi. Notice that in this case no rule corresponds to the axiom 5.

We now have all the necessary elements to show the first link between two kinds of modal calculi: we are going to prove that multiple sequents can be simulated by display sequents. In order to do this, we firstly define a translation $\delta$ from well-formed formulas and multisets of (well-formed) formulas of the language $\mathcal{L}_{-}^{\square}$ to, respectively, well-formed formulas and structures of the language of display logic. We have:

$$
\begin{aligned}
& -(\emptyset)^{\delta}=\left\{\begin{array}{c}
\text { if } \emptyset \text { is the antecedent, I } \\
\text { if } \emptyset \text { is the consequent, } I^{*}
\end{array}\right. \\
& -(\alpha)^{\delta}=\alpha \\
& -(-\alpha)^{\delta}=\alpha^{*} \\
& -\left(M^{*}\right)^{\delta}=\left(M^{\delta}\right)^{*}
\end{aligned}
$$

Given the translation $\delta$, we can define the translation $\tau$ in the following way:

$$
(\alpha, M \underset{n}{\longrightarrow} N, \beta)^{\tau}=\bullet^{n}\left((\alpha)^{\delta} \circ(M)^{\delta}\right) \Rightarrow(N)^{\delta} \circ(\beta)^{\delta}
$$

where $\bullet^{n} M$ stands for: $\overbrace{\bullet, \ldots, \bullet}^{n} M$.

We remark that, when $n=0$, which is to say, when the sequent is classical, we have:

$$
(\alpha, M \Rightarrow N, \beta)^{\tau}=(\alpha)^{\delta} \circ(M)^{\delta} \Rightarrow(N)^{\delta} \circ(\beta)^{\delta}
$$


From now on we shall write the name of a calculus for the system $\mathbf{K}$ indexed by the symbol “*," e.g. Dsk*, to denote any extension of that calculus with the special structural rules. We are now in a position to prove the following theorem.

Theorem 3.1. Let $M \underset{n}{\Rightarrow} N$ be any sequent of the calculi $\mathbf{M s k}^{*}$. Then every derivation of $M \square \vec{n}$ in $\boldsymbol{M s k}^{*}$ can be translated into a derivation of $(M \square \vec{n}$ $N)^{\tau}$ in $\boldsymbol{D} s \boldsymbol{k}^{*}$.

Proof. The proof is by induction on the derivation of the sequent $M \square \vec{n}$ in $\mathrm{Msk}^{*}$.

If $M \underset{n}{\Rightarrow} N$ is an axiom, then its translation $\tau$ is an admissible axiom of the form $\alpha \Rightarrow \alpha$ in display calculi.

If $M \square \vec{n}$ has been inferred by one of the structural rules or one of the shifting rules or one of the logical rules for the connective $\neg$, then the procedure is straightforward.

If $M \square \square_{n} N$ has been inferred by the logical rule that introduces the symbol $\wedge$ on the right side of the sequent (for the rule that introduces the symbol $\wedge$ on the left side of the sequent the procedure is analogous but easier), then we have:

$$
\begin{aligned}
& \frac{M \underset{n}{\rightrightarrows} N, \alpha \quad P \underset{n}{\Rightarrow} Q, \beta, \beta}{M, P \underset{n}{\rightleftarrows} N, Q, \alpha \wedge \beta} \wedge K
\end{aligned}
$$

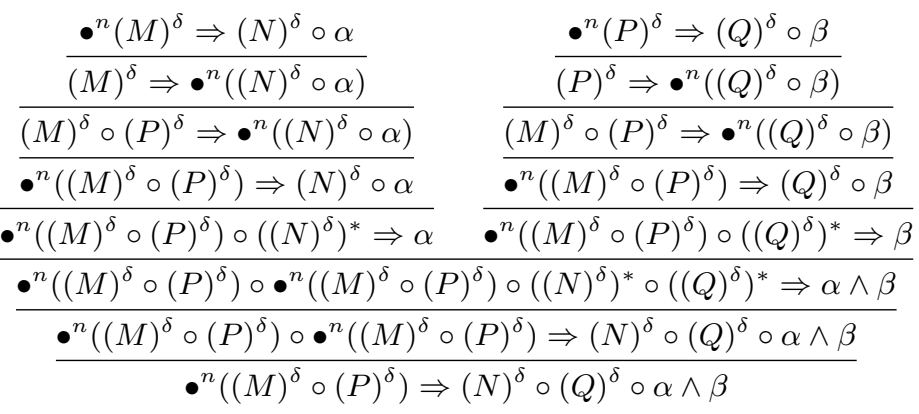

If $M \square \underset{n}{\Rightarrow} N$ is of the form $\square \alpha, M \square \underset{1}{\Rightarrow} N$ and has been inferred by the rule $\square A_{1}$, then we have:

$$
\frac{M \underset{1}{\square} N,-\alpha}{\square \alpha, M \underset{1}{\square}{ }^{\square}} \quad \rightsquigarrow
$$




$$
\begin{gathered}
\frac{\frac{\alpha \Rightarrow \alpha}{\square \alpha \Rightarrow \bullet \alpha}}{\square \alpha \circ(M)^{\delta} \Rightarrow \bullet \alpha} \quad \frac{\bullet(M)^{\delta} \Rightarrow(N)^{\delta} \circ(\alpha)^{*}}{\alpha \Rightarrow(N)^{\delta} \circ\left(\bullet(M)^{\delta}\right)^{*}} \\
\frac{\bullet\left(\square \alpha \circ(M)^{\delta}\right) \Rightarrow \alpha}{\frac{\bullet\left(\square \alpha \circ(M)^{\delta}\right) \Rightarrow(N)^{\delta} \circ\left(\bullet(M)^{\delta}\right)^{*}}{\bullet(M)^{\delta} \Rightarrow(N)^{\delta} \circ\left(\bullet\left(\square \alpha \circ(M)^{\delta}\right)\right)^{*}}} \\
\frac{\frac{(M)^{\delta} \Rightarrow \bullet\left((N)^{\delta} \circ\left(\bullet\left(\square \alpha \circ(M)^{\delta}\right)\right)^{*}\right)}{\square \alpha \circ(M)^{\delta} \Rightarrow \bullet\left((N)^{\delta} \circ\left(\bullet\left(\square \alpha \circ(M)^{\delta}\right)\right)^{*}\right)}}{\frac{\bullet\left(\square \alpha \circ(M)^{\delta}\right) \Rightarrow(N)^{\delta} \circ\left(\bullet\left(\square \alpha \circ(M)^{\delta}\right)\right)^{*}}{\bullet\left(\square \alpha \circ(M)^{\delta}\right) \circ \bullet\left(\square \alpha \circ(M)^{\delta}\right) \Rightarrow(N)^{\delta}}} \\
\frac{\bullet\left(\square \alpha \circ(M)^{\delta}\right) \Rightarrow(N)^{\delta}}{\bullet}
\end{gathered}
$$

If $M \underset{n}{\vec{n}} N$ is of the form $\square \alpha \square \vec{n} \Rightarrow N$ and has been inferred by the rule $\square A_{2}$, then we have:

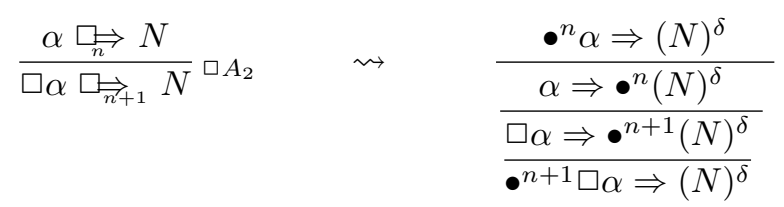

If $M \square \underset{n}{\square} N$ is of the form $M \stackrel{n}{n} \square \alpha$ and has been inferred by the rule $\square K$, then we have:

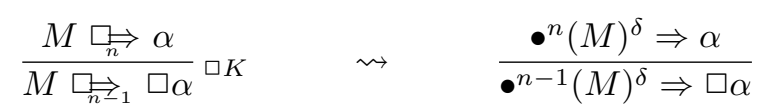

We finally analyze each of the cases in which $M \square \rightleftarrows N$ has been inferred by one of the special structural rules $d, t, b, 4$. We have:

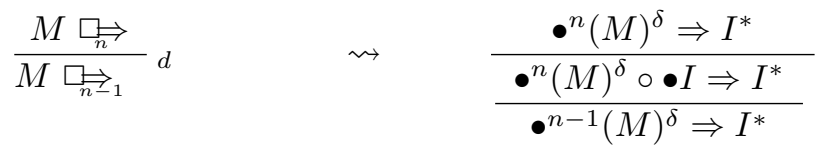

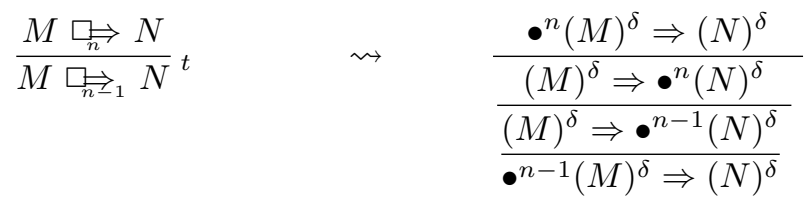




$$
\begin{aligned}
& \frac{B(M) \underset{n}{\Rightarrow} N}{B(M) \overrightarrow{n+1}_{1} N} 4 \quad \rightsquigarrow \quad \frac{\bullet^{n}(B(M))^{\delta} \Rightarrow(N)^{\delta}}{(B(M))^{\delta} \Rightarrow \bullet^{n}(N)^{\delta}} \\
& \frac{\frac{(B(M))^{\delta} \Rightarrow \bullet^{n+1}(N)^{\delta}}{\bullet^{n+1}(B(M))^{\delta} \Rightarrow(N)^{\delta}}}{{ }^{\prime}} \\
& \frac{M \underset{n}{\square} \stackrel{\rightrightarrows}{\Rightarrow} N_{n}^{*} M^{*}}{N^{*}} \\
& \frac{\frac{\bullet^{n}(M)^{\delta} \Rightarrow(N)^{\delta}}{\bullet^{n}(M)^{\delta} \Rightarrow\left((N)^{\delta}\right)^{* *}}}{\frac{(M)^{\delta} \Rightarrow \bullet^{n}\left(\left((N)^{\delta}\right)^{* *}\right)}{\frac{\left(\bullet^{n}\left(\left((N)^{\delta}\right)^{* *}\right)\right)^{*} \Rightarrow\left((M)^{\delta}\right)^{*}}{\bullet^{n}\left(\left((N)^{\delta}\right)^{*}\right) \Rightarrow\left((M)^{\delta}\right)^{*}}}}
\end{aligned}
$$

\section{Higher-arity Sequent Calculi and Display Cal- culi}

The idea of increasing the arity of a sequent has been firstly introduced by Schroeter (1955), and then further explored by Rousseau (1967) and Gottwald (1989). This idea was born as a natural solution to the problem of the lack of a sequent calculus for the Lukasiewicz $n$-valued logics. Indeed if two-place sequents were adequate to formalise two truth-values logics, then $n$-place sequents would have been suitable for formalising $n$-valued logics. More precisely we have that a classical sequent $M \Rightarrow N$ is true if, and only if, at least one of the $M$ 's is false or at least one of the $N$ 's is true. The n-valued sequent $M_{0}, M_{1}, \ldots, M_{n-1}$ for a $n$-valued logic is true if, and only if, there is a $j \leq n$ such that at least one of the $M_{j}$ 's has the value $j$.

This intuition has been recently taken up and adapted to modal logic. In this case one considers 4-place sequents in which the "two new truth values" are the necessarily true and the possibly false; that is to say, the 4-place sequent $M_{0}, M_{1}, M_{2}, M_{3}$ is true if, and only if, at least one of the $M_{0}$ is true or at least one of the $M_{1}$ is false or at least one of the $M_{2}$ is necessarily true or at least one of the $M_{3}$ is possibly false. Sato (1977) has been the first to apply the higher-arity sequent method to modal logic but he only obtained a calculus for the system S5. Blamey and Humberstone (1991) have constructed calculi with, first, 3-arity sequents, and, then, with 4-arity sequents, for the several systems of modal logic. Here we will refer to their work.

Higher-arity sequent. Given four WMF multisets $M, N, S$ and $T$, a higherarity sequent is an object of the following form:

$$
M \Rightarrow{ }_{S}^{T} N
$$

Obviously, if $S=T=\emptyset$, a higher-arity sequent becomes a classical sequent.

Classical and Higher-arity Structural Rules. In the higher-arity cal- 
Figure 8: Higher-arity and Classical Structural Rules

$$
\begin{aligned}
& \alpha \Rightarrow \emptyset_{\emptyset}^{\emptyset} \alpha \quad \frac{M \Rightarrow_{S}^{T} N}{\alpha, M \Rightarrow_{S}^{T} N} W A \quad \frac{M \Rightarrow_{S}^{T} N}{M \Rightarrow_{S}^{T} N, \alpha} W K \frac{\alpha, \alpha, M \Rightarrow_{S}^{T} N}{\alpha, M \Rightarrow_{S}^{T} N} C A \\
& \frac{M \Rightarrow_{S}^{T} N, \alpha, \alpha}{M \Rightarrow_{S}^{T} N, \alpha} C K \quad \frac{M \Rightarrow_{S}^{T} N}{M \Rightarrow_{S, \alpha}^{T} N} W_{n} A \quad \frac{M \Rightarrow_{S}^{T} N}{M \Rightarrow_{S}^{T, \alpha} N} W_{n} K \frac{M \Rightarrow_{S, \alpha, \alpha}^{T} N}{M \Rightarrow_{S, \alpha}^{T} N} C_{n} A \\
& \frac{M \Rightarrow_{S}^{T, \alpha, \alpha} N}{M \Rightarrow_{S}^{T, \alpha} N} C_{n} K \quad \frac{M \Rightarrow_{\emptyset}^{\emptyset} \alpha}{\emptyset \Rightarrow_{M}^{\alpha} \emptyset} r n \\
& \frac{\alpha, M \Rightarrow_{S}^{T} N \quad M \Rightarrow_{S}^{T} N, \alpha}{M \Rightarrow_{S}^{T} N} C u t_{\alpha}^{1} \quad \frac{M \Rightarrow_{S, \alpha}^{T} N \quad M \Rightarrow_{S}^{T, \alpha} N}{M \Rightarrow_{S}^{T} N} C u t_{\alpha}^{2}
\end{aligned}
$$

culi we have the classical structural rules of weakening and contraction, and the classical cut-rule, but also structural rules of a higher-arity type (see Figure 8). These structural rules simply reproduce the operations of weakening, contraction and cut at the higher-level.

Logical and Modal Rules. The logical rules for the symbols $\neg$ and $\wedge$ are straightforward adaptations of the classical rules to the higher-arity case. This means, more exhaustively, that even if the sequent might be a higher-arity sequent, the propositional rules act as in the classical case, e.g. the rules that introduces the symbol $\wedge$ on the right side of the sequent, is:

$$
\frac{M \Rightarrow_{S}^{T} N, \alpha \quad P \Rightarrow{ }_{Z}^{W} Q, \beta}{M, P \Rightarrow{ }_{S, Z}^{T, W} N, Q, \alpha \wedge \beta} \wedge K
$$

What we might call 'the correspondent of this rules for the higher-arity case,' i.e. the rule:

$$
\frac{M \Rightarrow_{S}^{T, \alpha} N \quad P \Rightarrow_{Z}^{W, \beta} Q}{M, P \Rightarrow_{S, Z}^{T, W, \alpha \wedge \beta} N, Q} \wedge_{n} K
$$

is an admissible rule.

We do not have modal rules, but the following modal axioms:

$$
(\square A) \quad \square \alpha \Rightarrow_{\emptyset}^{\alpha} \emptyset \quad(\square K) \quad \emptyset \Rightarrow_{\alpha}^{\emptyset} \square \alpha
$$

Modularity. The axioms, the structural rules, the logical and modal rules compose the calculus Hsk for the basic modal system K. In order to obtain the calculi for the other modal systems, we just add the appropriate special structural rule(s) (see Figure 9), as we have already explained for the display calculi. Notice that even in this case no rule corresponds to the axiom 5 . 
Figure 9: Special Structural Rules

$$
\frac{M \Rightarrow_{\emptyset}^{\emptyset} \emptyset}{\emptyset \Rightarrow_{M}^{\emptyset} \emptyset} d \quad \emptyset \Rightarrow_{\alpha}^{\emptyset} \alpha t \quad \frac{S \Rightarrow_{S}^{\emptyset} \alpha \quad M \Rightarrow_{\alpha, S^{\prime}}^{\emptyset} N}{M \Rightarrow_{S, S^{\prime}}^{\emptyset} N} 4 \quad \frac{S \Rightarrow_{\emptyset}^{N} \alpha \quad M \Rightarrow_{\alpha, S}^{T} N}{M \Rightarrow_{S}^{T} N} b
$$

We now have all the necessary elements to show the second link between two kinds of modal calculi: we are going to prove that higher-arity sequents can be simulated by display sequents. We firstly define the translation $\tau$ in the following way:

$$
\left(M \Rightarrow_{S}^{T} N\right)^{\tau}=\bigwedge \square S \circ M \Rightarrow \bigcirc \bullet T \circ N
$$

where $\bigcirc \bullet T$ should be seen as an analogous of $\bigwedge \square S$ : each formula belonging to $T$ is preceded by the symbol $\bullet$ and linked to the others by the symbol $\circ$. Informally the translation $\tau$ can be explained in the following way: all the formulas of the higher-arity sequent are linked by the symbol $\circ$, except the ones belonging to the multiset $S$ that becomes a conjunction of boxed formulas. Moreover each of the formulas belonging to the multiset $T$ is preceded by the symbol $\bullet$. Note that if $T \equiv N \equiv \emptyset$, then we have:

$$
\left(M \Rightarrow{ }_{S}^{\emptyset} \emptyset\right)^{\tau}=\bigwedge \square S \circ M \Rightarrow I^{*}
$$

By contrast if $S \equiv M \equiv \emptyset$, then we have:

$$
\left(\emptyset \Rightarrow_{\emptyset}^{T} N\right)^{\tau}=I \Rightarrow \bigcirc \bullet T \circ N
$$

We are now in a position to prove the following theorem. ${ }^{2}$

Theorem 4.1. Let $\mathcal{R} \subseteq\{d, t\}$ and $M \Rightarrow{ }_{T}^{S} N$ be any sequent of the calculi $\boldsymbol{H s k}$ $+\mathcal{R}$. Then every derivation of $M \Rightarrow{ }_{T}^{S} N$ in $\boldsymbol{H s k}+\mathcal{R}$ can be translated into a derivation of $\left(M \Rightarrow_{T}^{S} N\right)^{\tau}$ in $\boldsymbol{D} \boldsymbol{s} \boldsymbol{k}+\mathcal{R}$.

Proof. The proof is by induction on the derivation of the sequent $M \Rightarrow{ }_{S}^{T} N$ in Hsk $+\mathcal{R} .^{3}$

If $M \Rightarrow{ }_{S}^{T} N$ is an axiom, then its translation $\tau$ is an admissible axiom of the form $\alpha \Rightarrow \alpha$ in display calculi.

If $M \Rightarrow{ }_{S}^{T} N$ has been inferred by one of the classical or higher-arity rules of weakening, or by the classical rules of contraction, or by one of the logical rules for the connectives $\neg$ and $\wedge$, then the procedure is straightforward.

If $M \Rightarrow{ }_{S}^{T} N$ has been inferred by the rule $C_{n} A$ (for the rule $C_{n} K$ the procedure is analogous but easier), then we have:

$$
\frac{M \Rightarrow{ }_{S, \alpha, \alpha}^{T} N}{M \Rightarrow{ }_{S, \alpha}^{T} N} C_{n} A
$$




$\frac{\square \alpha \wedge \square \alpha \wedge \bigwedge \square S \circ M \Rightarrow \bigcirc \bullet T \circ N}{\square \alpha \wedge \square \alpha \wedge \bigwedge \square S \Rightarrow \bigcirc \bullet T \circ N \circ M^{*}}$
$\frac{\square \alpha \wedge \square \alpha \circ \wedge \square S \Rightarrow \bigcirc \bullet T \circ N \circ M^{*}}{\square \alpha \wedge \square \alpha \Rightarrow \bigcirc \bullet T \circ N \circ M^{*} \circ(\bigwedge \square S)^{*}}$
$\frac{\square \alpha \circ \square \alpha \Rightarrow \bigcirc \bullet T \circ N \circ M^{*} \circ(\bigwedge \square S)^{*}}{\square \alpha \Rightarrow \bigcirc \bullet T \circ N \circ M^{*} \circ(\bigwedge \square S)^{*}}$
$\frac{\square \alpha \circ \wedge \square S \Rightarrow \bigcirc \bullet T \circ N \circ M^{*}}{\square \alpha \wedge \bigwedge \square S \Rightarrow \bigcirc \bullet T \circ N \circ M^{*}}$
$\square \alpha \wedge \bigwedge \square S \circ M \Rightarrow \bigcirc \bullet T \circ N$

Note that in the second inference from the top, as well as in the fourth inference from the top, we are exploiting the fact that in display calculi logical rules are invertible; more precisely we are using the invertibility of the rule $\wedge A$.

If $M \Rightarrow_{S}^{T} N$ is of the form $\square \alpha \Rightarrow_{\emptyset}^{\alpha} \emptyset$, i.e. it is the modal axiom ( $\left.\square A\right)$, then we have:

$$
\frac{\alpha \Rightarrow \alpha}{\square \alpha \Rightarrow \bullet \alpha}
$$

If $M \Rightarrow{ }_{S}^{T} N$ is of the form $\emptyset \Rightarrow_{\alpha}^{\emptyset} \square \alpha$, i.e. it is the modal axiom ( $\left.\square K\right)$, then its translation $\tau$ is an admissible axiom of the form $\square \alpha \Rightarrow \square \alpha$ in display calculi.

If $M \Rightarrow_{S}^{T} N$ is of the form $\emptyset \Rightarrow_{M}^{\alpha} \emptyset$, and has been derived by the necessitation rule, i.e.

$$
\frac{M \Rightarrow_{\emptyset}^{\emptyset} \alpha}{\emptyset \Rightarrow_{M}^{\alpha} \emptyset}
$$

then we have a quite long proof to deal with and we develop it in the following several steps. Suppose that $M \equiv \gamma_{1}, \ldots, \gamma_{n}$. Then if we apply the translation $\tau$ on the premise of the necessitation rule, we obtain $\gamma_{1} \circ \ldots \circ \gamma_{n} \Rightarrow \alpha$ and then we can derive:

$$
\frac{\gamma_{1} \circ \ldots \circ \gamma_{n} \Rightarrow \alpha}{\frac{\gamma_{1} \wedge \ldots \wedge \gamma_{n} \Rightarrow \alpha}{\square\left(\gamma_{1} \wedge \ldots \wedge \gamma_{n}\right) \Rightarrow \bullet \alpha}}
$$

Let us then observe these $n$ analogous proofs:

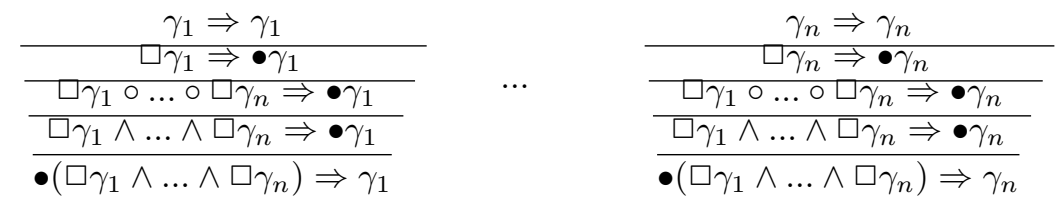

By applying the rule $\wedge K$ on the $n$ premises $\bullet\left(\square \gamma_{1} \wedge \ldots \wedge \square \gamma_{n}\right) \Rightarrow \gamma_{1} \ldots \bullet\left(\square \gamma_{1} \wedge\right.$ $\left.\ldots \wedge \square \gamma_{n}\right) \Rightarrow \gamma_{n}, n$-times, we obtain $\bullet\left(\square \gamma_{1} \wedge \ldots \wedge \square \gamma_{n}\right) \circ \ldots \circ \bullet\left(\square \gamma_{1} \wedge \ldots \wedge \square \gamma_{n}\right) \Rightarrow$ $\gamma_{1} \wedge \ldots \wedge \gamma_{n}$. Then we can proceed in the following way: 


$$
\frac{\bullet\left(\square \gamma_{1} \wedge \ldots \wedge \square \gamma_{n}\right) \circ \ldots \circ \bullet\left(\square \gamma_{1} \wedge \ldots \wedge \square \gamma_{n}\right) \Rightarrow \gamma_{1} \wedge \ldots \wedge \gamma_{n}}{\frac{\bullet\left(\square \gamma_{1} \wedge \ldots \wedge \square \gamma_{n}\right) \Rightarrow \gamma_{1} \wedge \ldots \wedge \gamma_{n}}{\square \gamma_{1} \wedge \ldots \wedge \square \gamma_{n} \Rightarrow \square\left(\gamma_{1} \wedge \ldots \wedge \gamma_{n}\right)}}
$$

Let us notice that what we have just proven is that the sequent $\bigwedge(\square M) \Rightarrow$ $\square(\bigwedge M)$ is derivable in display calculi. We can now apply a cut and reach our conclusion:

$$
\frac{\square \gamma_{1} \wedge \ldots \wedge \square \gamma_{n} \Rightarrow \square\left(\gamma_{1} \wedge \ldots \wedge \gamma_{n}\right) \quad \square\left(\gamma_{1} \wedge \ldots \wedge \gamma_{n}\right) \Rightarrow \bullet \alpha}{\square \gamma_{1} \wedge \ldots \wedge \square \gamma_{n} \Rightarrow \bullet \alpha}
$$

If $M \Rightarrow{ }_{S}^{T} N$ has been inferred by the rule $C u t_{\alpha}^{2}$ (for the rule $C u t_{\alpha}^{1}$ the procedure is analogous but easier), then we have:

$$
\frac{M \Rightarrow_{S, \alpha}^{T} N \quad M \Rightarrow_{S}^{T, \alpha} N}{M \Rightarrow_{S}^{T} N} C u t_{\alpha}^{2} \rightsquigarrow
$$

$$
\begin{aligned}
& \frac{\square \alpha \wedge \bigwedge \square S \circ M \Rightarrow \bigcirc \bullet T \circ N}{\square \alpha \circ \bigwedge \square S \circ M \Rightarrow \bigcirc \bullet T \circ N} \\
& \frac{\bigwedge \square S \circ M \Rightarrow \bullet \alpha \circ \bigcirc \bullet T \circ N}{(\bigcirc \bullet T \circ N)^{*} \circ \bigwedge \square S \circ M \Rightarrow \bullet \alpha} \\
& \square \alpha \circ \bigwedge \square S \circ M \Rightarrow \bigcirc \bullet T \circ N \\
& \bar{\bullet}\left((\bigcirc \bullet T \circ N)^{*} \circ \bigwedge \square S \circ M\right) \Rightarrow \alpha \\
& (\bigcirc \bullet T \circ N)^{*} \circ \bigwedge \square S \circ M \Rightarrow(\square \alpha)^{*} \quad(\square \alpha)^{*} \Rightarrow \bigcirc \bullet T \circ N \circ(\bigwedge \square S \circ M)^{*} \\
& (\bigcirc \bullet T \circ N)^{*} \circ \bigwedge \square S \circ M \Rightarrow \neg \square \alpha \quad \neg \square \alpha \Rightarrow \bigcirc \bullet T \circ N \circ(\bigwedge \square S \circ M)^{*} \\
& \frac{(\bigcirc \bullet T \circ N)^{*} \circ \bigwedge \square S \circ M \Rightarrow \bigcirc \bullet T \circ N \circ(\bigwedge \square S \circ M)^{*}}{\frac{\bigwedge \square S \circ M \circ \bigwedge \square S \circ M \Rightarrow \bigcirc \bullet T \circ N \circ \bigcirc \bullet T \circ N}{\bigwedge \square S \circ M \Rightarrow \bigcirc \bullet T \circ N}}
\end{aligned}
$$

We finally analyse the cases in which $M \Rightarrow_{S}^{T} N$ has been inferred by one of the special structural rules $d, t$. We have:

$$
\begin{array}{ll}
M \Rightarrow_{\emptyset}^{\emptyset} \emptyset \\
\emptyset \Rightarrow \Rightarrow_{M}^{\emptyset} \emptyset
\end{array}
$$

Notice that we could use the sequent $\bigwedge(\square M) \Rightarrow \square(\bigwedge M)$, since we have already shown that it is a derivable sequent in display calculi. 


$$
\emptyset \Rightarrow_{\alpha}^{\emptyset} \alpha \quad t \quad \rightsquigarrow \quad \frac{\alpha \Rightarrow \alpha}{\square \alpha \Rightarrow \bullet \alpha}
$$

$\otimes$

\section{Tree-hypersequent Calculi and Display Cal- culi}

The basic idea of the tree-hypersequent calculi is to reproduce in the framework of the sequent calculus the structure of the tree-frames of Kripke semantics. This is done by: (i) considering, as in the hypersequent case, $n$ sequents at a time; (ii) introducing two new meta-linguistic symbols: the slash (/) and the semicolon (;). Given that the several sequents should be seen as worlds of a treeframe of Kripke semantics, the slash should be understood as the accessibility relation of a tree-frame of Kripke semantics, e.g. if two sequents $M \Rightarrow N$ and $S \Rightarrow T$ are separated by a slash in the following way $M \Rightarrow N / S \Rightarrow T$, this should be read as: either the sequent $M \Rightarrow N$ is true at a world $x$, or the sequent $S \Rightarrow T$ is true at a world $y$ such that $x R y$. The semicolon, by contrast, serves to reproduce in the framework of the sequent calculus the fact that in a tree-frame of Kripke semantics $n$ different worlds $y_{1}, \ldots, y_{n}$ can all be related, by the accessibility relation, to a world $x$. For example, if we have the structure $M \Rightarrow N / S \Rightarrow T ; Z \Rightarrow W$, this should be read as: either the sequent $M \Rightarrow N$ is true at a world $x$, or the sequent $S \Rightarrow T$ is true at a world $y_{1}$ such that $x R y_{1}$, or the sequent $Z \Rightarrow W$ is true at a world $y_{2}$ such that $x R y_{2}$.

Tree-hypersequents were firstly introduced by Kashima (1994) (see also Hasuo and Kashima (2003), Ishigaki and Kikuchi (2007)) with a different name and a different notation. Then they have successively been considered by Brunnler (2006), who uses the same notation of Kashima, and Poggiolesi (2008b) and Poggiolesi (2008a). Here we consider this last work.

Syntactic Notation. We shall use $\Gamma, \Delta, \ldots$ to denote sequents (SEQ), $G, H$, ... to denote tree-hypersequents (THS), and $\underline{X}, \underline{Y}, \ldots$ to denote finite multisets of tree-hypersequents (MTHS).

Tree-hypersequent and Its Interpretation. The notion of tree-hypersequent is inductively defined in the following way:

- if $\Gamma \in \mathrm{SEQ}$, then $\Gamma \in \mathrm{THS}$,

- if $\Gamma \in \mathrm{SEQ}$ and $\underline{X} \in \mathrm{MTHS}$, then $\Gamma / \underline{X} \in$ THS.

Given this definition, we have that an example of a tree-hypersequent is an object of the following form:

$$
\Delta_{1} /\left(\Delta_{2} / \Delta_{3}\right) ;\left(\Delta_{4} /\left(\Delta_{5} / \Delta_{6}\right) ; \Delta_{7}\right)
$$

The intended interpretation of a tree-hypersequent is: 
Figure 10: Axioms and Logical Rules of the calculus Thsk

$$
\begin{aligned}
& G[p, M \Rightarrow N, p] \quad \frac{G[M \Rightarrow N, \alpha]}{G[\neg \alpha, M \Rightarrow N]} \neg A \quad \frac{G[\alpha, M \Rightarrow N]}{G[M \Rightarrow N, \neg \alpha]} \neg K \quad \frac{G[\alpha, \beta, M \Rightarrow N]}{G[\alpha \wedge \beta, M \Rightarrow N]} \wedge A \\
& \frac{G[M \Rightarrow N, \alpha] \quad G[M \Rightarrow N, \beta]}{G[M \Rightarrow N, \alpha \wedge \beta]} \wedge K
\end{aligned}
$$

Figure 11: Modal Rules

$$
\frac{G[\square \alpha, M \Rightarrow N / \alpha, S \Rightarrow T]}{G[\square \alpha, M \Rightarrow N / S \Rightarrow T]} \square A \quad \frac{G[M \Rightarrow N / \square \alpha \Rightarrow \alpha ; \underline{X}]}{G[M \Rightarrow N, \square \alpha / \underline{X}]} \square K
$$

Figure 12: Special Logical Rules

$$
\begin{aligned}
& \frac{G[\square \alpha, M \Rightarrow N / \alpha \Rightarrow ; \underline{X}]}{G[\square \alpha, M \Rightarrow N / \underline{X}]}{ }_{d} \quad \frac{G[\square \alpha, \alpha, M \Rightarrow N]}{G[\square \alpha, M \Rightarrow N]} t \quad \quad \frac{G[\square \alpha, M \Rightarrow N][\alpha, S \Rightarrow T]}{G[\square \alpha, M \Rightarrow N][S \Rightarrow T]} 4 \\
& \frac{G[\square \alpha, \alpha, M \Rightarrow N / S \Rightarrow T]}{G[M \Rightarrow N / \square \alpha, S \Rightarrow T]} b \quad \frac{G[\alpha, M \Rightarrow N][\square \alpha, S \Rightarrow T]}{G[M \Rightarrow N][\square \alpha, S \Rightarrow T]} 5 \\
& -(M \Rightarrow N)^{\tau}:=\bigwedge M \rightarrow \bigvee N \\
& -\left(\Gamma / G_{1} ; \ldots ; G_{n}\right)^{\tau}:=\Gamma^{\tau} \vee \square G_{1}^{\tau} \vee \ldots \vee \square G_{n}^{\tau}
\end{aligned}
$$

In order to display the rules of the calculi, we will use the notation $G[*]$ to refer to a tree-hypersequent $G$ together with one hole [*], where the hole should be understood, metaphorically, as a zoom by means of which we can focus attention on a particular point, $*$, of $G$. Such an object becomes a real tree-hypersequent whenever the symbol $*$ is appropriately replaced by (i) a sequent $\Gamma$; in this case we will write $G[\Gamma]$ to denote the tree-hypersequent $G$ together with a specific occurrence of a sequent $\Gamma$ in it; or (ii) two sequents, $\Gamma / \Sigma$, one after another and separated by a slash; in this case we will write $G[\Gamma / \Sigma]$ to denote the treehypersequent $G$ together with a specific occurrence of a sequent $\Gamma$ immediately followed by a specific occurrence of a sequent $\Sigma$; or (iii) a tree-hypersequent $H$; in this case we will write $G[H]$ to denote the tree-hypersequent $G$ together with a specific occurrence of a tree-hypersequent $H$ in it.

We will also use the notation $G[\Gamma][\Sigma]$ to denote the tree-hypersequent $G$ together with a specific occurrence of a sequent $\Gamma$ and, successive to it (thinking the treehypersequent as a tree-frame, successive means that it is in the same branch but $n$ worlds-sequents further on), a specific occurrence of the sequent $\Sigma$.

Structural Rules. A nice feature of the tree-hypersequent calculi is that it can be proved that all the structural rules are (height-preseving) admissible in them (Poggiolesi (2008b)). The (height-preseving) admissible structural rules of 
the tree-hypersequent calculi are not only the well known rules of weakening and contraction, but there are also external structural rules, i.e. rules that operate on the structure of the tree-hypersequent. An example of an (height-preseving) admissible external structural rule is the rule of merge that has the form:

$$
\frac{G\left[\Delta /(M \Rightarrow N / \underline{X}) ;\left(P \Rightarrow Q / \underline{X^{\prime}}\right) ; \underline{Y}\right]}{G\left[\Delta /\left(M, P \Rightarrow N, Q / \underline{X} ; \underline{X^{\prime}}\right) ; \underline{Y}\right]} \text { merge }
$$

We also emphasise that a special structural rule corresponds to each special logical rule and it is admissible in those calculi where the correspondent special logical rule is present.

Logical and Modal Rules. Let us first of all remark that the logical rules of the tree-hypersequent calculi (see Figure 10) are the classical ones adapted to the case of tree-hypersequents. More precisely, these rules say that we can apply the classical inference rules to any sequent belonging to the tree-hypersequent.

The logical and the modal rules are provable to be (height-preserving) invertible without any use of the cut-rule.

Lack of Modularity. Simply the axioms and the logical and modal rules compose the calculus Thsk for the basic modal system K. In order to obtain the calculi for the other modal systems, we just add the appropriate special logical rule(s) (see Figure 12). We note two important facts: (i) each time that the two rules 4 and $b$ are added to the calculus Thsk, the rule 5 should be added too; (ii) the rule 5 fails to reflect the expressive power of the axiom 5 in the framework of the sequent calculus: e.g., if this rule is added to the calculus Thsk we do not obtain an adequate and cut-free tree-hypersequent calculus for the system K5. Therefore this rule can only be used in addition to the rules 4 and $b$.

We also point out that in order to show the deep link between tree-hypersequent calculi and display calculi, we have taken the rules 4 and 5 in an different form from that used in Poggiolesi (2008b).

We finally have all the necessary elements to show the third link between syntactic modal calculi: we are going to prove that tree-hypersequents can be simulated by display sequents. In order to prove this result, let us start by introducing the following translations, which are quite similar to the ones introduced by Mints (1997):

$$
\begin{aligned}
& \left(\alpha_{1}, \ldots, \alpha_{n} \Rightarrow \beta_{1}, \ldots, \beta_{m}\right)^{s}=\alpha_{1} \circ \ldots \circ \alpha_{n} \Rightarrow \beta_{1} \circ \ldots \circ \beta_{m} \\
& \left(M_{1}, \ldots, M_{n} \Rightarrow N_{1}, \ldots, N_{m}\right)^{p}=\left(M_{1}\right)^{*} \circ \ldots \circ\left(M_{n}\right)^{*} \circ N_{1} \circ \ldots \circ N_{m}
\end{aligned}
$$

In other words, the translation $s$ allows one to substitute the comma with the symbol $\circ$; while the translation $p$ allows one to transform, by moving the antecedent in the appropriate way, two-side sequents into one-side sequents.

$$
\text { For } G \equiv M \Rightarrow N / H_{1} ; \ldots ; H_{n} \text {, set: }
$$




$$
(G)^{s}=\left(M \Rightarrow N \circ \bullet\left(H_{1}\right)^{s p} \circ \ldots \circ \bullet\left(H_{n}\right)^{s p}\right)^{s}
$$

Lemma 5.1. Given a tree-hypersequent $G[H]$ in its translation $(G[H])^{s}$, we can always rewrite the tree-hypersequent $H$ in the following way:

$$
R \Rightarrow(H)^{s p}
$$

where $R$ is some display structure that depends on $G$.

Proof. By induction on the complexity of the form of the tree-hypersequent $G[H]$.

If $G[H] \equiv H$, then the procedure is straightforward.

If $G[H] \equiv M \Rightarrow N / H ; H_{1} ; \ldots ; H_{m}$, then we have:

$$
\frac{M \Rightarrow N \circ \bullet(H)^{s p} \circ \bullet\left(H_{1}\right)^{s p} \circ \ldots \circ \bullet\left(H_{m}\right)^{s p}}{\left(N \circ \bullet\left(H_{1}\right)^{s p} \circ \ldots \circ \bullet\left(H_{m}\right)^{s p}\right)^{*} \circ M \Rightarrow \bullet(H)^{s p}}
$$

If finally $G[H] \equiv M \Rightarrow N / G^{\prime}[H] ; H_{1} ; \ldots ; H_{m}$, then we have $M \Rightarrow N \circ$ - $\left(G^{\prime}[H]\right)^{s p} \circ \bullet\left(H_{1}\right)^{s p} \circ \ldots \circ \bullet\left(H_{m}\right)^{s p}$. By the inductive hypothesis on $G^{\prime}[H]$, we obtain $\left(\bullet\left(G^{\prime}\right)^{s p}\right)^{*} \circ M \Rightarrow N \circ(H)^{s p} \circ \bullet\left(H_{1}\right)^{s p} \circ \ldots \circ \bullet\left(H_{m}\right)^{s p}$, and from this we easily obtain $\left(N \circ \bullet\left(H_{1}\right)^{s p} \circ \ldots \circ \bullet\left(H_{m}\right)^{s p}\right)^{*} \circ\left(\bullet\left(G^{\prime}\right)^{s p}\right)^{*} \circ M \Rightarrow(H)^{s p} . \quad$

Theorem 5.2. Let $G[H]$ be any tree-hypersequent of the calculi Thsk*. Then every derivation of $G[H]$ in Thsk $^{*}$ can be translated into a derivation of $R \Rightarrow$ $(H)^{s p}$, where $R$ is some display structure that depends on $G$, in $\boldsymbol{D} s \boldsymbol{k}^{*}$.

Proof. The proof is by induction on the derivation of the sequent $G[H] .{ }^{4}$

If $G[H]$ is an axiom, then $R \Rightarrow(H)^{s p}$ is derivable in $\mathbf{D} \mathbf{s k}^{*}$ by several applications of the external and classical rules of weakening on admissible axioms of the form $\alpha \Rightarrow \alpha$.

If $G[H]$ has been inferred by one of the logical rules for the connectives $\neg$ and $\wedge$, then the procedure is straightforward.

If $G[H]$ has been inferred by the modal rule $\square K$, then we have:

$$
\begin{aligned}
& \frac{G[M \Rightarrow N / \Rightarrow \alpha ; \underline{X}]}{G[M \Rightarrow N, \square \alpha / \underline{X}]} \square K \\
& \frac{R \Rightarrow M^{*} \circ N \circ \bullet(\underline{X})^{s p} \circ \bullet\left(I^{*} \circ \alpha\right)}{\frac{\left(M^{*} \circ N \circ \bullet(\underline{X})^{s p}\right)^{*} \circ R \Rightarrow \bullet\left(I^{*} \circ \alpha\right)}{\bullet\left(\left(M^{*} \circ N \circ \bullet(\underline{X})^{s p}\right)^{*} \circ R\right) \Rightarrow I^{*} \circ \alpha}} \\
& \frac{\bullet\left(\left(M^{*} \circ N \circ \bullet(\underline{X})^{s p}\right)^{*} \circ R\right) \Rightarrow \alpha}{\left(M^{*} \circ N \circ \bullet(\underline{X})^{s p}\right)^{*} \circ R \Rightarrow \square \alpha} \\
& R \Rightarrow \square \alpha \circ M^{*} \circ N \circ \bullet(\underline{X})^{s p}
\end{aligned}
$$


If $G[H]$ has been inferred by the modal rule $\square A$, then we have: ${ }^{5}$

$$
\begin{aligned}
& \frac{G[\square \alpha, M \Rightarrow N / \alpha, S \Rightarrow T]}{G[\square \alpha, M \Rightarrow N / S \Rightarrow T]} \square A \quad \rightsquigarrow \\
& R \Rightarrow \square \alpha^{*} \circ A \circ \bullet\left(\alpha^{*} \circ B\right) \\
& \overline{\left(\square \alpha^{*} \circ A\right)^{*} \circ R \Rightarrow \bullet\left(\alpha^{*} \circ B\right)} \\
& \text { • }\left(\left(\square \alpha^{*} \circ A\right)^{*} \circ R\right) \Rightarrow \alpha^{*} \circ B \\
& B^{*} \circ \bullet\left(\left(\square \alpha^{*} \circ A\right)^{*} \circ R\right) \Rightarrow \alpha^{*} \\
& \alpha \Rightarrow\left(B^{*} \circ \bullet\left(\left(\square \alpha^{*} \circ A\right)^{*} \circ R\right)\right)^{*} \\
& \square \alpha \Rightarrow \bullet\left(B^{*} \circ \bullet\left(\left(\square \alpha^{*} \circ A\right)^{*} \circ R\right)\right)^{*} \\
& \text { - } \square \alpha \Rightarrow\left(B^{*} \circ \bullet\left(\left(\square \alpha^{*} \circ A\right)^{*} \circ R\right)\right)^{*} \\
& B^{*} \circ \bullet\left(\left(\square \alpha^{*} \circ A\right)^{*} \circ R\right) \Rightarrow(\bullet \square \alpha)^{*} \\
& \bullet\left(\left(\square \alpha^{*} \circ A\right)^{*} \circ R\right) \Rightarrow(\bullet \square \alpha)^{*} \circ B \\
& \overline{\left(\square \alpha^{*} \circ A\right)^{*} \circ R \Rightarrow \bullet\left((\bullet \square \alpha)^{*} \circ B\right)} \\
& \square \alpha \circ\left(\square \alpha^{*} \circ A\right)^{*} \circ R \Rightarrow \bullet\left((\bullet \square \alpha)^{*} \circ B\right) \\
& \text { - }\left(\square \alpha \circ\left(\square \alpha^{*} \circ A\right)^{*} \circ R\right) \Rightarrow(\bullet \square \alpha)^{*} \circ B \\
& \bullet \square \alpha \Rightarrow\left(\bullet\left(\square \alpha \circ\left(\square \alpha^{*} \circ A\right)^{*} \circ R\right)\right)^{*} \circ B \\
& \square \alpha \Rightarrow \bullet\left(\left(\bullet\left(\square \alpha \circ\left(\square \alpha^{*} \circ A\right)^{*} \circ R\right)\right)^{*} \circ B\right) \\
& \square \alpha \circ\left(\square \alpha^{*} \circ A\right)^{*} \circ R \Rightarrow \bullet\left(\left(\bullet\left(\square \alpha \circ\left(\square \alpha^{*} \circ A\right)^{*} \circ R\right)\right)^{*} \circ B\right) \\
& \text { • }\left(\square \alpha \circ\left(\square \alpha^{*} \circ A\right)^{*} \circ R\right) \Rightarrow\left(\bullet\left(\square \alpha \circ\left(\square \alpha^{*} \circ A\right)^{*} \circ R\right)\right)^{*} \circ B \\
& \bullet\left(\square \alpha \circ\left(\square \alpha^{*} \circ A\right)^{*} \circ R\right) \circ \bullet\left(\square \alpha \circ\left(\square \alpha^{*} \circ A\right)^{*} \circ R\right) \Rightarrow B \\
& \bullet\left(\square \alpha \circ\left(\square \alpha^{*} \circ A\right)^{*} \circ R\right) \Rightarrow B \\
& \square \alpha \circ\left(\square \alpha^{*} \circ A\right)^{*} \circ R \Rightarrow \bullet B \\
& \left(\square \alpha^{*} \circ A\right)^{*} \circ R \Rightarrow \square \alpha^{*} \circ \bullet B \\
& R \Rightarrow \square \alpha^{*} \circ \square \alpha^{*} \circ A \circ \bullet B \\
& R \Rightarrow \square \alpha^{*} \circ A \circ \bullet B
\end{aligned}
$$

If $G[H]$ has been inferred by the special logical rule $t$, then we have:

$$
\begin{aligned}
& \frac{G[\square \alpha, \alpha, M \Rightarrow N]}{G[\square \alpha, M \Rightarrow N]} t \\
& \frac{R \Rightarrow \square \alpha^{*} \circ \alpha^{*} \circ M^{*} \circ N}{\frac{\alpha \Rightarrow R^{*} \circ \square \alpha^{*} \circ M^{*} \circ N}{\square \alpha \Rightarrow \bullet\left(R^{*} \circ \square \alpha^{*} \circ M^{*} \circ N\right)}} \\
& \frac{\square \alpha \Rightarrow R^{*} \circ \square \alpha^{*} \circ M^{*} \circ N}{\square \alpha \circ \square \alpha \Rightarrow R^{*} \circ M^{*} \circ N} \\
& \frac{\square \alpha \Rightarrow R^{*} \circ M^{*} \circ N}{R \Rightarrow \square \alpha^{*} \circ M^{*} \circ N}
\end{aligned}
$$


If $G[H]$ has been inferred by the special logical rule $b$, then we have:

$$
\begin{aligned}
& \frac{G[\alpha, M \Rightarrow N / \square \alpha, S \Rightarrow T]}{G[M \Rightarrow N / \square \alpha, S \Rightarrow T]} b{ }^{\frac{R \Rightarrow \alpha^{*} \circ M^{*} \circ N \circ \bullet\left(\square \alpha^{*} \circ S^{*} \circ T\right)}{\left(M^{*} \circ N \circ \bullet\left(\square \alpha^{*} \circ S^{*} \circ T\right)\right)^{*} \circ R \Rightarrow \alpha^{*}}} \\
& \frac{\frac{\alpha \Rightarrow\left(\left(M^{*} \circ N \circ \bullet\left(\square \alpha^{*} \circ S^{*} \circ T\right)\right)^{*} \circ R\right)^{*}}{\square \alpha \Rightarrow \bullet\left(\left(M^{*} \circ N \circ \bullet\left(\square \alpha^{*} \circ S^{*} \circ T\right)\right)^{*} \circ R\right)^{*}}}{\frac{\left.\left(\bullet\left(M^{*} \circ N \circ \bullet\left(\square \alpha^{*} \circ S^{*} \circ T\right)\right)^{*} \circ R\right)^{*}\right)^{*} \Rightarrow \square \alpha^{*}}{\bullet\left(\left(M^{*} \circ N \circ \bullet\left(\square \alpha^{*} \circ S^{*} \circ T\right)\right)^{*} \circ R\right) \Rightarrow \square \alpha^{*}}} \\
& \frac{\bullet\left(\left(M^{*} \circ N \circ \bullet\left(\square \alpha^{*} \circ S^{*} \circ T\right)\right)^{*} \circ R\right) \Rightarrow \square \alpha^{*} \circ S^{*} \circ T}{\left(M^{*} \circ N \circ \bullet\left(\square \alpha^{*} \circ S^{*} \circ T\right)\right)^{*} \circ R \Rightarrow \bullet\left(\square \alpha^{*} \circ S^{*} \circ T\right)} \\
& \frac{R \Rightarrow \bullet\left(\square \alpha^{*} \circ S^{*} \circ T\right) \circ M^{*} \circ N \circ \bullet\left(\square \alpha^{*} \circ S^{*} \circ T\right)}{R \Rightarrow M^{*} \circ N \circ \bullet\left(\square \alpha^{*} \circ S^{*} \circ T\right)}
\end{aligned}
$$

If $G[H]$ has been inferred by the special logical rule 4 , then we use the same procedure adopted for the rule $\square A$ plus we exploit the special display structural rule 4.

If $G[H]$ has been inferred by the special logical rule 5 , then we use the same procedure adopted for the rule $b$ plus we exploit the special display structural rule $4 . \quad \square$

\section{Conclusions}

As we have seen in the previous sections, display calculi have great expressive power: in them the new structural symbols are directly applied on the formulas, and classical operations are decomposed into more primitive operations so that each inferential step is shown. As Wansing (1998, p.187) says, we can look at the display method as a "background theory" where we can "compare with each other various kinds of generalised sequent systems," and which can be applied to various types of logic, not only the modal ones. On the other hand the display calculi's strength comes with several disadvantages. The proofs are complicated, the question of the lack of the subformula property arises (see Avron (1996) and Wansing (2002)), and, as Kracht (1996) has proved, it is undecidable whether or not a display calculus is decidable. Once again, quoting Wansing (1998, p.187), we can claim that "there is a price to be paid for a greater generality."

Analogous conclusions can be drawn concerning the calculus of structures mentioned above. As we have seen in the introduction, display calculi can be plugged into the calculus of structures, which therefore seems to represent a more general framework. This fact is also confirmed by the applicability of the 
method of structures to a broad number of different logics. On the other hand, in this case, the drawbacks are significant. Above all: we do not even have a direct proof of cut-elimination (the cut-elimination is proved indirectly via translations to other calculi). This seems a high price to pay for generality. Maybe we have gone too far away from the original sequent calculus. Let us thus return to the realm of more self-contained generalisations of the sequent calculus and ask the following question: if one is willing to give up the powerful generality of the display calculi in order to get those results which are not obtainable in the display framework, do any of the other syntactic methods that we have presented in this paper represent a valid alternative? In order to answer this question, let us examine each of these methods in turn. The higher-arity method should be the first to be ruled out since the calculi are not cut-free. On the other hand, even the multiple sequent calculi should be discarded because of some negative features that they present: the cut-rule is not formulated, they lack a syntactic proof of cut-elimintion and a proof of decidability. Hence the tree-hypersequent method seems to be our last possibility. In this case we are not disappointed: indeed the tree-hypersequent calculi are cut-free (and this can be proved in a syntactic and in a semantic way), they enjoy the subformula property, the structural rules are (height-preserving) admissible, the logical rules are (heightpreserving) invertible and they are decidable (once again, this can be proved in a syntactic and in a semantic way). In a word, they have the properties that the display calculi lack and vice-versa. We can therefore claim that the tree-hypersequents method represents a good alternative to the display method and that we should appreciate this alternative since choice is often an enviable privilege.

\section{Notes}

\footnotetext{
${ }^{1}$ Notice that, in order to shorten the proofs in the calculi $\mathbf{D} \mathbf{s k}^{*}$, we may use several rules in a row and indicate them with just one inference.

${ }^{2}$ As the reader can easily check the Theorem 4.1 does not hold for the structural rules 4 and $b$.

${ }^{3}$ As in the previous theorem, in order to shorten the proofs in the calculi Dsk $+\mathcal{R}$, we may use several rules in a row and indicate them with just one inference.

${ }^{4}$ Notice that, as usual, in order to shorten the proofs in the calculi Dsk*, we may use several rules in a row and indicate them with just one inference.

${ }^{5}$ For the sake of clarity, in the following proofs in display calculi, we are going to denote the structure $M^{*} \circ N$ with the letter A, and the structure $S^{*} \circ T$ with the letter B.
}

\section{References}

Avron, A. (1996). The method of hypersequents in the proof theory of propositional non - classical logic. In Hodges, W., Hyland, M., Steinhorn, C., and Strauss, J., editors, Logic: from foundations to applications, pages 1-32. Oxford University Press, Oxford.

Belnap, N. D. (1982). Display logic. Journal of Philosophical Logic, 11:375-417. 
Belnap, N. D. (1990). Linear logic displayed. Notre Dame Journal of Formal Logic, 31:14-25.

Belnap, N. D. (1996). The display problem. In Wansing, H., editor, Proof Theory of Modal Logic. Kluwer Academic Publisher, Dordrecht.

Blamey, S. and Humberstone, L. (1991). A perspective on modal sequent logic. Publications of the Research Institute for Mathematical Sciences, Kyoto University, 27:763-782.

Brunnler, K. (2006). Deep sequent systems for modal logic. Advances in Modal Logic AiML, 6:107-119.

Curry, H. B. (1952). The elimination theorem when modality is present. Journal of Symbolic Logic, 17:249-265.

Fitting, M. (1983). Proof Methods for Modal and Intuitionistic Logics. Reidel, Dordrecht.

Goré, R. (1998). Substructural logics on display. Logic Journal of the IGPL, 6:669-694.

Goré, R. and Tiu, A. (2007). Classical Modal Display Logic in the Calculus of Structures and Minimal Cut-free Deep Inference Calculi for S5. Journal of Logic and Computation, 17:767-794.

Gottwald, S. (1989). Mehrwertige Logik. Akademie-Verlag, Berlin.

Guglielmi, A. (2007). A system of interaction and structure. ACM Transactions on computational Logic, 8:1-64.

Hasuo, I. and Kashima, R. (2003). Kripke completeness of First-Order Constructive Logics with Strong Negation. Logic Journal of the IGPL, 11:615-646.

Indrezejczak, A. (1997). Generalised sequent calculus for propositional modal logics. Logica Trianguli, 1:15-31.

Ishigaki, R. and Kikuchi, K. (2007). Tree-sequent method for subintuitionistic predicate logics. Proceedings of the 16th International Conference on Automated Reasoning with Analytic Tableaux and Related Methods, LNAI, 4548, 149-164.

Kashima, R. (1994). Cut-free sequent calculi for some tense logics. Studia Logica, 53:119-135.

Kracht, M. (1996). Power and weakness of the modal display calculus. In Wansing, H., editor, Proof Theory of Modal Logic. Kluwer Academic Publishers., Dordrecht.

Mints, G. (1997). Indexed systems of sequents and cut-elimination. Journal of Philosophical Logic, 26:671-696. 
Poggiolesi, F. (2008a). The method of tree-hypersequent for modal propositional logic. To appear in Towards Mathematical Philosophy, Studia Logica Library.

Poggiolesi, F. (2008b). Sequent calculi modal logic. Ph.D Thesis, pages 1-224.

Restall, G. (1998). Displaying and deciding substructural logics. I: Logics with contraposition. Journal of Philosophical Logic, 27:179-216.

Rousseau, G. (1967). Sequents in many-valued logic. I. Fundamenta Mathematicae, 60:23-131.

Sato, M. (1977). A study of kripke-type models for some modal logics by gentzen's sequential method. Publications of the Research Institute for Mathematical Sciences, Kyoto University, 13:381-468.

Schroeter, M. (1955). Methoden zur axiomatisierung beliebiger aussagen- und praedikatenkalkuele. Zeitschrift fuer mathematische Logik und Grundlagen der Mathematik, 1:214-251.

Stewart, C. and Stouppa, P. (2005). A systematic proof theory for several modal logics. In Advances in Modal Logic, Vol 5, pages 309-333. Kings College Publications, ?.

Wansing, H. (1994). Sequent systems for normal modal propositional logics. Journal of Logic and Computation, 4:125-142.

Wansing, H. (1997). Displaying as temporalizing. sequent systems for subintuitionistic logics. In Hakama, S., editor, Logic, Language and Computation. Kluwer Academic Publishers., Dordrecht.

Wansing, H. (1998). Displaying Modal Logic. Kluwer Academic Publisher, Dordrecht.

Wansing, H. (2002). Sequent systems for modal logics. In Handbook of Philosophical Logic, Vol 8, pages 61-145. Kluwer, Dordrecht.

Zeman, J. (1973). Modal logic. The Lewis-Modal Systems. Oxford University Press, Oxford. 